Fecha de recepción: 10/11/2014

Palabras clave: investigación, comprensión, práctica, experiencia, director

Keywords: research, comprehension, practice, experience, school principal
Reflexiones sobre la concepción de subjetividad en una investigación sobre directores desde el enfoque clínico

\section{Reflections upon the concept of subjectivity in a research project regarding headmasters from the clinical approach}

\section{Lorena Sanchez Troussel}

Instituto de Investigaciones en Ciencias de la Educación, Facultad de Filosofía y Letras, Universidad de Buenos Aires/Consejo Nacional de Investigaciones Científicas y Técnicas, Argentina lorenalbs@hotmail.com

\begin{abstract}
Resumen
Las reflexiones en el campo de la psicología y la filosofía contemporánea en torno a la cuestión del sujeto y la subjetividad, han movilizado durante los años 2013 y 2014, intercambios dentro del equipo de investigación del Programa La Clase Escolar, del Instituto de Investigaciones en Ciencias de la Educación [IICE], de la Facultad de Filosofía y Letras de la Universidad de Buenos Aires. El presente artículo plantea las modificaciones en la formulación del objeto de investigación, producto de las reflexiones sobre la subjetividad, de un proyecto que indaga el trabajo de directores de escuela en contextos de vulnerabilidad social en la Ciudad de Buenos Aires. Se describe el proceso que da cuenta de cómo el objeto se transforma: desde el estudio de las prácticas de directores al estudio de la experiencia, en función del cambio
\end{abstract}


conceptual sujeto-subjetividad.

En el primer apartado se presenta una síntesis de los intereses que llevaron a la formulación del proyecto de investigación sobre directores y se explica el porqué de la adopción del enfoque clínico en la investigación. En segundo lugar se hace referencia a la primera formulación del objeto de investigación a partir de la noción de prácticas. En el tercer apartado presentamos la reformulación del objeto a partir de la noción de experiencia. Nos detenemos aquí en analizar algunos rasgos de la concepción de subjetividad que la noción de experiencia adoptada pone de relieve. Por último analizamos los límites y posibilidades que la adopción de la noción de experiencia presenta en la investigación sobre directores.

Reflections in the field of psychology and contemporary philosophy around the question of the subject and subjectivity have mobilized exchanges, during 2013 - 2014, within the research team of the "Program School Class", from Research Institute of Educational Sciences [IICE], where the project we will account for here belongs. This article discusses the changes in the formulation of the research object of a project that explores the work of school principals in contexts of social vulnerability in the City of Buenos Aires, due to a series of reflections on subjectivity. It is described the process that accounts for the transformation of the research object: from the study of the school principals' practices to the study of the principals' experience, following the conceptual change from the construct of subject to that one of subjectivity.

The first section summarizes the interests that led to the formulation of the research project on principals and explain the reason for the adoption of the clinical approach. In second place we refer to the first formulation of the research object with the construct of practices. In the third 
section we present the reformulation of the object from the notion of experience and we analyze some features of the construct of subjectivity that the notion of experience highlights. Finally we analyze the limits and possibilities in the research on principals that the adoption of the notion of experience poses.

\section{El interés por el estudio de directores de escuela}

Como hemos mencionado, describiremos en este trabajo algunos de los procesos de reflexión acerca de la construcción del objeto de estudio de una investigación en curso. Se trata de un proyecto de tesis doctoral iniciado en el año 2012, con sede en el Instituto de Investigaciones en Ciencias de la Educación [IICE] de la Universidad de Buenos Aires [UBA]. El título del proyecto de tesis es: "Dirigir una escuela en contextos de vulnerabilidad social. Estudio de la experiencia de directores de escuelas de barrios urbano-marginados". Se trata de una investigación de tipo cualitativa realizada desde el enfoque clínico en sentido amplio (Souto, 2010). Comprende el estudio de dos casos: dos directores de escuela primaria de la Ciudad de Buenos Aires que trabajan en escuelas que reciben a alumnos de barrios urbano-marginados.

Los principales intereses iniciales puestos en juego en la definición y redefinición del objeto de estudio fueron:

- el estudio del trabajo de directores de escuela, es decir de la función directiva;

- la escuela en contextos de vulnerabilidad social.

El propósito del proyecto de investigación del que aquí hablaremos, es el de comprender los rasgos que asume la experiencia directiva en los mencionados contextos. Se busca que dicha producción permita: reflexionar acerca de las necesidades de formación de los directores que trabajan en estos contextos y de los rasgos que la formación debiera tener.

El interés por comprender el hacer de los directores, con el fin de pensar su formación en futuros proyectos, nos llevó a adoptar un enfoque 
de investigación acorde, que nos permitiera -sin desconocer problemáticas de índole social-, poder considerar aspectos vinculados con la subjetividad, es decir los modos singulares en que cada director cumple esa función en cada una de las instituciones. El interés por estudiar la singularidad de cada director, en su contexto, en la temporalidad, en el marco de la institución y sus características; nos llevó a la adopción del enfoque clínico en sentido amplio (Souto, 2010). El mismo se constituyó en opción epistemológica-metodológica, en tanto:

Se trata de un modo de abordaje, de una mirada que permite acercarse al otro (objeto de investigación) desde una actitud de receptividad, de porosidad que facilita la comunicación no sólo consciente sino inconsciente con el otro, un gesto profesional caracterizado por la receptividad, la apertura a los significados, pensamientos, efectos, representaciones imaginarias, que permiten tomar en uno- en el sentido de contención psíquica- los contenidos conscientes e inconscientes, manifiestos y latentes del otro, sus modos peculiares de expresión, sus silencios, sus resistencias y defensas, su manera de presentarse y construir su ethos en la situación intersubjetiva de una entrevista o una observación (Souto, 2010, p. 63).

La consonancia entre los intereses de investigación definidos y el enfoque clínico adoptado supuso la realización de un estudio de seguimiento de cada director en terreno, en profundidad, en la temporalidad y en las situaciones de interacción en cada institución. El sujeto investigador se incluye en las observaciones y entrevistas desde impresiones subjetivas que quedan registradas en el protocolo y que permiten el análisis posterior de la implicación.

Es decir, que la adopción del enfoque clínico en el estudio de directores permite:

- Conocer al director en su medio, en el contexto institucional y así poder dar cuenta del modo en que se vincula con los alumnos, los docentes, el equipo de dirección, la comunidad, la tarea desde aspectos manifiestos y latentes.

- Dar cuenta de las transformaciones, las dificultades, el sufrimiento 
que el director vive en el ejercicio del rol en los mencionados contextos. - Comprender la experiencia directiva desde miradas múltiples (multirreferencialidad teórica) que permiten interpretarla en sus dimensiones psíquica, instrumental, institucional y social.

\section{La primera formulación del objeto de investigación: prácticas de directores}

Partiendo del interés por el estudio de los directivos en contextos de vulnerabilidad social, la formulación inicial del objeto se centró en el concepto de prácticas. El proyecto de investigación se propuso la descripción y el análisis de las prácticas de directores de escuelas de barrios urbano-marginados.

El modo de concebir las prácticas en este momento se articuló en torno de tres concepciones sobre la misma:

1) La idea de práctica en términos de un hacer orientado por valores (concepción aristotélica de la práctica):

\footnotetext{
Hablar de la educación como práctica se refiere no sólo a su acepción más usual, un hacer, sino que representa, en su sentido aristotélico, una preocupación moral, un guiarse por valores y fines que la acción busca realizar en sí misma; pero esos mismos valores no cobran un significado fijo y externo a la acción, es en la propia acción por donde acaban por adquirir significado (Contreras y Pérez, 2010, p.12).
}

2) La idea de práctica como un proceso humano que supone la transformación de la realidad (concepción de tipo antropológico e instrumental): "[la práctica es un] proceso de transformación de una realidad en otra realidad y que requiere de la intervención de un operador humano (sujeto)" (Barbier, 1996, citado en Blanchard Laville y Fablet, 1996, p. 31). En esta definición, una práctica más que la identificación de los resultados, supone la identificación de los procesos de transformación de la realidad, así como de los componentes que entran en juego de modo directo en la producción de esos resultados. 
3) La idea de práctica en tanto práctica profesional (concepción que pone de relieve la dimensión sociológica de la práctica):

por práctica entenderemos cualquier forma coherente y compleja de actividad humana cooperativa, establecida socialmente, mediante la cual se realizan los bienes inherentes a la misma, mientras se intenta lograr los modelos de excelencia que le son apropiados a esa forma de actividad (MacIntyre, 1987, p. 233).

A partir de la reflexión teórica sobre las nociones de sujeto y subjetividad realizadas en el grupo de estudios dentro del equipo y, de los significados que surgieron en los contactos con el terreno en la investigación sobre directores, nos planteamos algunos interrogantes. Los mismos estuvieron relacionados con:

- La concepción de subjetividad: ¿qué concepción de subjetividad subyace a la noción de prácticas?

- El modo en que el director se vincula con la realidad de la escuela: ¿Cómo el director/a otorga sentido a su tarea en un contexto complejo e incierto en el que el mismo ejercicio del rol directivo entra en cuestión? ¿Cómo otorga sentido a su tarea en un contexto social en el que la construcción de sentido sobre el futuro, la educación y la escuela misma, parecen estar puestos en duda? ¿Cómo la noción de práctica nos permite dar cuenta de estas problemáticas?

En este marco de inquietudes y preguntas, la concepción de práctica presentaba algunas limitaciones:

- En primer lugar, la idea de práctica supone una escisión entre sujeto y objeto. La práctica es el proceso por el cual un sujeto transforma la realidad (objeto). Algunas concepciones de práctica refieren, a su vez, a que el sujeto es transformado en el proceso de la práctica. Sin embargo, la separación de sujeto y objeto, incluso en las concepciones que refieren a una relación dialéctica entre ambos, es constitutiva de la noción.

- Por otra parte, la idea de práctica en tanto práctica profesional supone considerar centralmente la idea de profesión en tanto campo 
definido socialmente. Es decir que la consideración del concepto de práctica en la definición del objeto de investigación significa poner de relieve una dimensión sociológica del objeto, en tanto se trata de analizar las regulaciones y normas que guían la práctica directiva en tanto profesión. En este sentido, desde esta construcción teórica no se focaliza, a nuestro entender, en el modo singular en que ese director se vincula con su función, la escuela, el barrio, etc.

- En tercer lugar, consideramos que la idea de práctica supone colocar en primer plano el hacer, la acción (ya fuera en torno a valores, en torno a los componentes del proceso de transformación, como en torno a ese hacer y sus definiciones culturales y sociales) y no el plano del padecer. Este interés por el padecimiento y la posibilidad de su significación y re significación, es decir, la cuestión del sentido, se consideran centrales para pensar a los directores en un contexto en el que la realidad externa a la escuela (la pobreza) podría favorecer todavía más los procesos de alienación propios de las instituciones (Fernández, 1996).

\section{La reformulación del objeto de investigación: la expe- riencia de directores}

En el marco de las reflexiones teóricas sobre la noción de subjetividad en el grupo de investigación y de las preguntas a las que dieron lugar los primeros contactos con el terreno, se inició un camino hacia la redefinición del objeto de investigación. El propósito fue la construcción de un objeto desde categorías teóricas que nos permitieran considerar las problemáticas que hemos definido en el apartado anterior. Después de analizar distintas conceptualizaciones, definimos al objeto de investigación a partir de la noción de experiencia.

Si bien la misma es sumamente polisémica y compleja (Sanchez Troussel, 2012) y por ello su utilización crea importantes desafíos para la investigación empírica, a nuestro entender, permite abordar el estudio del trabajo de los directores en contextos de vulnerabilidad social desde la perspectiva que nos interesa. 
Sostenemos entonces que la noción de experiencia:

1- Permite poner en cuestión la escisión entre sujeto y objeto, propia de la concepción moderna de subjetividad y presente en la noción de prácticas.

2- Al poner en tensión la escisión sujeto-objeto, la noción de experiencia también interroga la concepción de la relación sujeto-mundo como una relación estrictamente epistemológica;

3- Sin descuidar el análisis de la dimensión del hacer del director, incorpora la dimensión del padecer. Es decir: la posibilidad de analizar aspectos de la subjetividad vinculados con el pathos.

Ahora bien ¿qué supone la concepción moderna de subjetividad? y, ¿en qué sentido la noción de experiencia la interroga?

En términos esquemáticos podemos señalar que la modernidad definió al sujeto como autofundado y en oposición al mundo. El mundo (la naturaleza y los otros) es lo que se opone al sujeto y lo que se opone al sujeto es ob-jeto (ob-jectum es lo que yace frente de...). En la concepción moderna, el sujeto se relaciona con el objeto a través de la representación, es decir: el sujeto se representa el mundo y lo lleva así al ámbito de su interioridad. La capacidad de representación del mundo es lo que le permite al sujeto conocerlo, esto es: dominarlo, controlarlo, ponerlo bajo el poder de su racionalidad (Frydman, 2012). El sujeto es, en la concepción moderna, dueño de la realidad (de la realidad externa cuando se representa el mundo y de la realidad interna cuando vuelve la mirada sobre sí).

En esta concepción de subjetividad, el yo accede a la conciencia de forma inmediata y esto es índice de transparencia epistemológica: el yo es tranparente a sí mismo, considera que las cosas pueden conocerse en sí mismas. En la concepción cartesiana, Dios sigue apareciendo como garante del conocimiento de las cosas, del mundo, pero es el hombre quien las conoce. La subjetividad es fundamento absoluto y a la vez lugar de ese fundamento absoluto:

Para Descartes, entonces, el yo es el sujeto del conocimiento, y ese 
yo es una substancia, una cosa, una res en el sentido aristotélico. La sustancia es lo que está por debajo, lo que subyace como núcleo terminado y completo, lo que tiene un ser en sí que no depende de otra cosa para ser [...] A partir de Descartes sabemos que las cosas son dudosas, que no son tal como aparecen. Sin embargo, el punto en que Descartes no duda es cuando afirma que la conciencia es tal y como parece a sí misma; en ella, sentido y conciencia del sentido coinciden (Frydman, 2012, p. 35).

El costo de este modo de comprender la subjetividad es el hiato entre el yo (pensamiento) y el mundo (realidad); la reducción del pensar a la actividad psíquica de consciencia (Frydman, 2012).

Entonces, ¿de qué modo la noción de experiencia discute estos rasgos de la subjetividad moderna?

Por una parte, en tanto la noción de experiencia (en desarrollos como los de Dewey, 2000) discute la dicotomía entre sujeto y objeto y critica la idea de sujeto autofundado. El pragmatismo deweyano hace hincapié en la defensa de una comprensión holística de la experiencia y se muestra en disgusto con las filosofías que pretenden descubrir los componentes esenciales de la realidad así como también de aquellas filosofías que buscan postular principios a priori. Dewey (2000) insiste en sus desarrollos sobre la "experiencia educativa", en afirmar la unidad entre sujeto y mundo.

La experiencia es para Dewey (2000) una noción que permite dar cuenta del intercambio entre el ser vivo y su medio físico y social. Este intercambio no es únicamente un asunto de conocimiento o epistemológico. Sostiene que en la experiencia se ponen en juego un conjunto de acciones, pero también de afecciones. De este modo, para Dewey, experiencia y pensamiento no son dos términos opuestos, sino que se reclaman mutuamente. En este sentido, la mirada holística sobre la experiencia, le permite al filósofo poner en tensión la relación dicotómica entre sujeto y objeto propia de la modernidad. A su vez, le permite cuestionar la idea de un sujeto autofundado y comprender la subjetividad no como un a priori, sino como eventual emergente. En este sentido, afirma que 
"la personalidad, el sí mismo y la subjetividad son funciones eventuales que emergen con la complejidad de interacciones organizadas, desde el punto de vista orgánico y social. La individualización personal tienen su sustento y condiciones en los simples sucesos" (Dewey, citado en González Monteagudo, 2002, p. 23).

De acuerdo con Jay (2009) conceptualizaciones de experiencia como la de Dewey presentan un nuevo modo de concebir la subjetividad. En los desarrollos previos al pragmatismo la experiencia supone un sujeto integrado, coherente y más o menos autónomo con capacidad para actuar en el mundo, sobre él. Para Jay, los pragmatistas como Dewey enriquecieron las concepciones sobre experiencia, aportando "una clave nueva y pos subjetiva a la discusión" (Jay, 2009, p. 28), en la que la oposición sujeto-mundo se relativiza:

Dado que [experiencia] se refiere tanto a lo que se está experimentando cuanto al proceso subjetivo de experimentarlo, la palabra suele operar como un término "paraguas" para superar la escisión epistemológica entre sujeto y objeto. Los pragmatistas norteamericanos eran proclives a utilizarlo de esta manera. Si sumamos a ello los adjetivos que habitualmente acompañan a la palabra, tales como "vivida", "interior" y "genuina", es fácil comprender por qué el término ha tenido una historia tan llamativa y continúa fascinando nuestra imaginación.

Hemos señalado hasta aquí que hablar de experiencia nos permite poner en cuestión el hiato sujeto-mundo propio de la concepción moderna de subjetividad. Nos permite, a su vez, caracterizar la relación sujeto-objeto más allá de su faceta epistemológica. Pero además, la noción de experiencia posibilita poner en duda la idea de un sujeto integrado, coherente y autónomo, con capacidad de actuar sobre el mundo. Ahora bien ¿qué supone hablar de un sujeto que no está integrado, que no es autónomo? Podríamos responder a esta pregunta desde marcos teóricos diversos. Señalaremos brevemente la respuesta que desde el psicoanálisis puede darse a dicha pregunta.

Frydman (2012) nos señala que el término sujeto arriba al psicoanálisis 
con Lacan, sin embargo, la teoría freudiana sin hablar estrictamente de sujeto, supone un fuerte cuestionamiento a la concepción moderna de subjetividad al cuestionar la identificación entre actividad psíquica y actividad consciente. Freud (citado en Frydman, 2012, p.42-43) señala:

Qué puede decir entonces el filósofo frente a una doctrina que, como el psicoanálisis, asevera que lo anímico es, más bien, en sí inconsciente, y la condición de inconsciente no es más que una cualidad que puede agregarse o no al acto anímico singular, y eventualmente, cuando falta, no altera más en éste? Dice, desde luego, que algo anímico inconsciente es un disparate, una contradicción in adjecto, y no quiere percatarse de que con este juicio no hace más que repetir su propia definición -acaso demasiado estrecha- de lo anímico.

La concepción freudiana saca al yo del lugar de amo en su propia casa y abre la concepción de una subjetividad que nunca se someterá a los dictados de la conciencia. El yo deja de ser amo para pasar a padecer los dictados del inconsciente. Pese a que los desarrollos del psicoanálisis tienen más de un siglo, la incorporación de sus aportes al campo de la enseñanza y la formación es todavía escasa. En el caso del estudio sobre directores, la utilización de enfoques o marcos que incorporen aportes del psicoanálisis son casi inexistentes y nos interesa incorporarlos en el estudio al que nos estamos refiriendo.

Nos detendremos en la idea de pathos y el modo en que la noción de experiencia la incorpora. Entre los antecedentes del concepto moderno de experiencia, suele incluirse la palabra griega pathos. Pathos significa "algo que sucede" (Jay, 2009, p.27), asociado al sentido de lo que uno sufre o soporta. En cierto modo podríamos decir que lo otro-inconsciente da cuenta de la dimensión pathémica de la subjetividad (en el sentido que los fenómenos inconscientes son a pesar del sujeto, es decir, escapan a su voluntad y control). Sin embargo presentaremos algunos otros significados relacionados con el pathos. Jay (2009, p. 27) señala al respecto: 
Cuando experiencia adquiere el sentido de experimento, sus dimensiones prácticas se activan, pero cuando se vincula al pathos - es decir, al hecho de que la experiencia puede sobrevenirnos sin buscarla o desearla- se destaca su dimensión pasiva. En este caso la paciencia puede convertirse en una virtud y la espera de un encuentro que uno no puede forzar se considera una fuente de experiencia.

Consideramos que la incorporación de esta dimensión de la experiencia resulta central, en tanto posibilita plantear interrogantes acerca de los modos en que cada director se relaciona con el contexto en el que trabaja (caracterizado por la vulnerabilidad y la exclusión social) y consigo mismo. Nos permite reflexionar acerca del modo en que la realidad de las escuelas los afecta. Los directores señalan que, en ocasiones, las situaciones que enfrentan se les presentan como inciertas y los desbordan, desbordan sus modos de pensar y de hacer. Entonces ¿qué sucede cuando la realidad se presenta de modo tal que los saberes disponibles no son permiten significarla y actuar sobre ella? ¿Qué sucede cuando esa dimensión propositiva de la experiencia parece quedar paralizada? Considerar estos problemas supone considerar el modo en que lo imprevisto, lo incierto se relaciona con la experiencia:

La experiencia tiene lugar como un acontecer del que nadie es dueño, que no está determinada por el peso propio de una u otra observación sino que en ella todo viene a ordenarse de una manera realmente impenetrable [...] La experiencia surge con esto o con lo otro, de repente, de improviso, y sin embargo no sin preparación, y vale hasta que aparezca una experiencia nueva (Gadamer, 1977, p. 28).

En síntesis, dentro de las conceptualizaciones disponibles de experiencia se consideraron para la reformulación del objeto de estudio aquellas que permiten:

- Poner en tensión la escisión sujeto-objeto propia de la concepción moderna de subjetividad.

- Concebir la relación sujeto-objeto como una relación compleja y no 
sólo como una relación epistemológica.

- Incorporar el análisis de las emociones y de los fenómenos inconscientes, como constitutivos de la relación sujeto-mundo.

- Comprender la subjetividad en términos de eventual emergente y no como un a priori.

- Estudiar las acciones de los directores pero también los modos en que la realidad los afecta; analizar el vínculo que establecen con la incertidumbre.

Nos interesan entonces las conceptualizaciones que conciben a la experiencia como espacio paradojal (Jay, 2009). Las mismas posibilitan, a nuestro entender, el estudio de la experiencia en la tensión objeto y sujeto, íntimo y público, singular y universal, propio del sujeto y propio del mundo, difuminando las barreras entre cada uno de los opuestos. Esta forma de concebir la experiencia supone que por más que se trate a la experiencia como una posesión personal, nunca lo es, en tanto ésta se adquiere en el encuentro con la otredad: "la experiencia [...] se halla en el punto nodal de la intersección entre el lenguaje público y la subjetividad privada, entre los rasgos comunes expresables y el carácter inefable de la interioridad individual" (Jay, 2009, p. 20).

La noción de experiencia, desde esta concepción, permite poner en primer plano la dimensión de la vivencia: cómo estos individuos concretos viven la dirección, como actúan pero también como padecen en esos contextos, en relación con su hacer, en el vínculo con los otros, con la institución, con el barrio; poniendo de relieve la idea de que el sujeto es siendo, y por lo tanto es en la experiencia. Así:

La experiencia resulta ser un campo de fuerza dinámico [...] ni totalmente dentro, ni totalmente fuera del Yo; un Yo que, de todas formas, nunca es previo a la experiencia y se niega a ser reducido tanto a sus modos trascendentales como a sus modos empíricos. Cargado con la energía del deseo, resiste la domesticación en los discursos convencionales, heteronormativos y procreativos de la sexualidad... Implica el lenguaje aunque lo excede; está disponible para la 
observación objetiva, aunque es una ficción producida después del hecho (Jay, 2009, p. 452).

A partir de las reflexiones aquí planteadas, el objeto de estudio queda enunciado como: La experiencia de directores de escuela de barrios urbano-marginados. La reformulación del objeto no supone un objeto de estudio distinto, en tanto nos sigue interesando el estudio de los directores en contextos de vulnerabilidad social. Sin embargo, como hemos intentado exponer, la redefinición teórica del objeto a partir de la noción de experiencia, hace posible el abordaje de nuevas facetas del mismo a la vez que otorga centralidad a dimensiones descuidadas bajo la formulación de prácticas. En síntesis, la reformulación del objeto de estudio supone, la consideración del ejercicio profesional de los directores a partir de considerar aquello que hacen y también aquello que padecen; supone el análisis de las dimensiones sociales y técnicas de la profesión, pero también de la dimensión psíquica de la misma.

\section{A modo de cierre}

La concepción moderna de subjetividad supone un sujeto autofundado, integrado y autónomo. El sujeto es yo y se define en oposición al mundo (objeto) y al que domina por la vía del conocimiento. La posibilidad del sujeto de representar el mundo y llevarlo así al mundo de su interioridad, significa la posibilidad de habitar en un mundo inteligible, luminoso, lógico, reconocible como propio; un mundo predecible y legible, pensable y controlable, como si fuera la propia conciencia la que lo construyera, la que lo creara, la que lo dominara según su propia unidad.

Las conceptualizaciones de experiencia que hemos presentado discuten la concepción moderna de subjetividad como terreno incontaminado. Por supuesto que el recorrido realizado no agota ni es expresión de la compleja discusión al respecto. Sin embargo, nos ha permitido puntualizar algunos aportes que movilizaron los cambios de orden teórico y epistemológico en nuestra investigación.

En síntesis, concebir la experiencia como campo de fuerzas dinámico 
(Jay, 2009), como espacio de tensión entre Yo y no-yo, permite acceder al modo particular en que un sujeto concreto, vivo, tal es el director, se vincula con el mundo y los otros y otorga sentido a la realidad que vive. Permite acercarnos a los sentidos que produce, sin desconocer que ese modo de significar le es a la vez propio y ajeno. A su vez, experiencia es una noción que, por su carácter complejo, controversial y finalmente inasible, entendemos nos permite estar alerta frente a la posibilidad de reducción de sentidos diversos en significados unívocos y estáticos. El pasaje de la práctica a la experiencia modifica el objeto de estudio y significa una búsqueda propia de la investigación para ajustar la definición y aproximación del objeto. El enfoque clínico en sentido amplio permite abordar la complejidad y singularidad de cada caso, de cada director en su entorno e incluir la lectura del psicoanálisis en su articulación con otras posibles. Queda todavía por considerar, a la luz de los resultados finales, una reflexión sistemática acerca de las metodologías de recolección de datos y de las técnicas de análisis más adecuadas en el estudio de la experiencia de los directivos desde las consideraciones aquí realizadas.

\section{Bibliografía}

Blanchard Laville, C., y Fablet, D. (1996). L'analyse des pratiques professsionnelles. París: PUF.

Contreras, J. y Pérez, L. (2010). Investigar la experiencia educativa. Madrid: Morata.

Dewey, J. (2000) Experiencia y Educación. México DF: Losada.

Fernández, L. (1994). Instituciones Educativas. Dinámicas institucionales en situaciones críticas. Buenos Aires: Paidós.

Frydman, A. (2012). La subversión de Lacan. Una introducción a la noción de sujeto. Buenos Aires: Ediciones Continente.

Gadamer, H.G. (1977). Verdad y Método I. Salamanca: Sígueme. 
Jay, M. (2009). Cantos de experiencia. Variaciones modernas sobre un tema universal. Buenos Aires: Paidós.

MacIntyre, A. (1987). Tras la virtud. Barcelona: Crítica.

Sanchez Troussel, L. y Renzi, M. (2012). La noción de experiencia en la investigación sobre trabajo y formación docente. Algunos interrogantes epistemológicos. Revista Educaçao e Politicas em debate. Vol. 2, № 2 , 304-324.

Souto, M. (2010). La investigación clínica en Ciencias de la Educación. Revista del Instituto de Investigaciones en Ciencias de la Educación, XVIII (29), 57-74. 